\title{
Hadron calorimeter module prototype for baryonic matter studies at Nuclotron
}

\author{
O.P. Gavrishchuk ${ }^{\dagger} \ddagger$, V.P. Ladygin ${ }^{\dagger}$, Yu.P. Petukhov ${ }^{\dagger}$ and S.Ya. Sychkov ${ }^{\dagger}$ \\ $\dagger$ Joint Institute for Nuclear Research, 141980 Dubna, Russia \\ ${ }^{\ddagger}$ E-mail: Oleg.gavrishchuk@cern.ch
}

\begin{abstract}
The prototype of the hadron calorimeter module consisting of 66 scintillator/lead layers with the $15 \times 15 \mathrm{~cm}^{2}$ cross section and $\sim 5$ nuclear interaction lengths has been designed and produced for the zero degree calorimeter of the BM@N experiment. The prototype has been tested with high energy muon beam of the U-70 accelerator at IHEP. The results of the beam test for different types of photo multipliers and light guides are presented. The results of the Monte-Carlo simulation of the calorimeter response and energy resolution are presented for the $2-16 \mathrm{GeV}$ protons.
\end{abstract}

PACS:29.40Vj- Calorimeters

\section{Introduction}

The study of the dense baryonic matter at Nuclotron (BM@N project) [1] is proposed as a first stage in the heavy-ion program at NICA [2]. The major direction in the research program of BM@N project is the production of strange matter in heavy-ion collisions at beam energies between 2 and $6 \mathrm{~A} \cdot \mathrm{GeV}[3]$. The other topics of the experimental program can be related with the study of in-medium effects for strange particles decaying in hadronic modes [4], hard probes and correlations [5], soft photons and neutral mesons [6], polarization effects [7] etc. For these purposes an experimental setup will be installed at the $6 \mathrm{~V}$ beamline in the fixed-target hall of the Nuclotron. The first results with the relativistic deuteron [8] and carbon [9] beams demonstrated the feasibility of these studies with light nuclei.

An important direction of the relativistic heavy-ion collisions studies is the measurements of different observables as a function of the centrality parameter. The concept of centrality is based on the use of the impact parameter value which possible to define as a distance between the centres of

\footnotetext{
${ }^{1}$ Corresponding author: vladygin@jinr.ru
} 
the colliding nuclei in the plane, perpendicular to the beam direction. Since the impact parameter is a non-measurable value, it is possible to use such characteristic of the collision, as the part of a total energy which is carried away by the spectators: protons, neutrons and nuclear fragments. Most of spectators has a small transverse momentum and is emitted in a narrow cone near the primary beam direction. To measure the spectators energy the calorimeter at zero angle, so-called Zero Degree Calorimeter (ZDC), is usually used. Another purpose of ZDC is the central events selection at the trigger level during data taking.
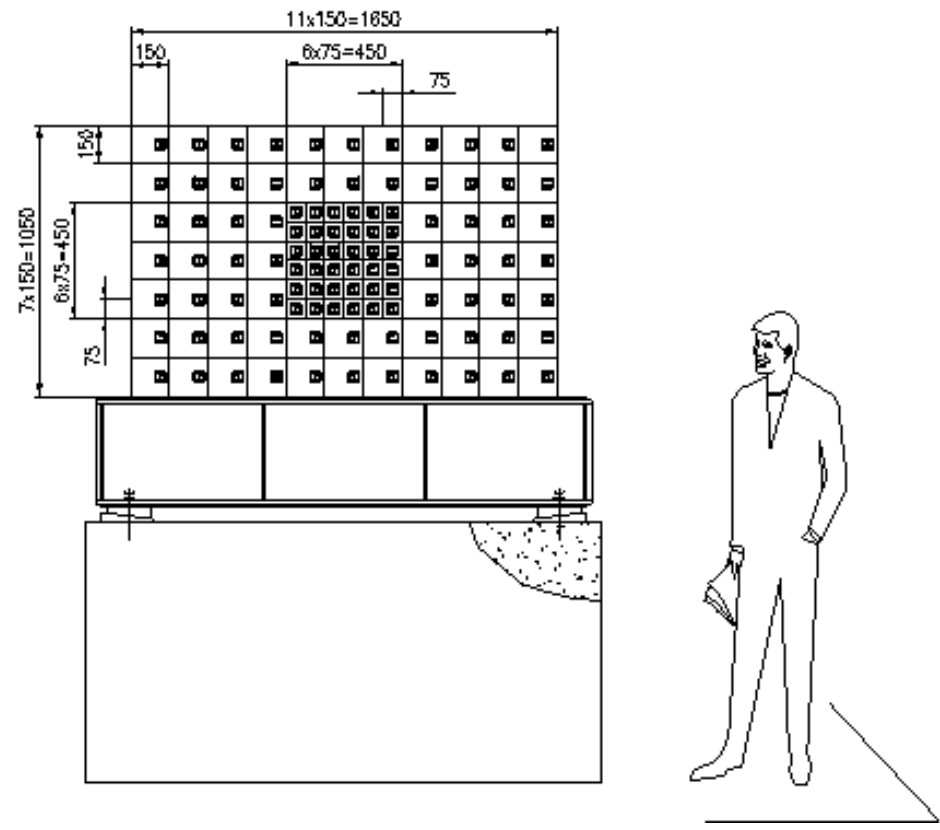

Figure 1: Transversal view of ZDC consisting of 104 HCAL modules. Central and peripheral parts and consist of $6 \times 6$ modules and 68 modules with cross section of $7.5 \times 7.5 \mathrm{~cm}^{2}$ and $15 \times 15 \mathrm{~cm}^{2}$, respectively.

Main ZDC option for the first stage of BM@N experiment [1] will be based on the scintillator/lead sandwich type of the hadron calorimeter (HCAL) used in WA98 [10] and COMPASS [11] experiments at CERN. Central part of ZDC consists of an array of $6 \times 6$ modules with the cross section of $7.5 \times 7.5$ $\mathrm{cm}^{2}$ each, while peripheral part contains 68 modules of $15 \times 15 \mathrm{~cm}^{2}$ transversal size. Such granularity allows one to operate with the nuclear beam intensities up to $1 \mathrm{MHz}$, which is sufficient for the first stage of the experiment. For the beam intensity of $10 \mathrm{MHz}$ it will be necessary to remove several central 
modules. The schematic view of the transverse cross section of ZDC for BM@N experiment is presented in Fig.1. The HCAL module [1] consists of 80 scintillator/lead layers with the sampling structure of $1 / 4$. This sampling is optimal to obtain the $e / \pi$ and $e / \mathrm{p}$ ratios close to 1 and the good energy resolution for all particles in the energy range of $2 \div 100 \mathrm{GeV}$ with $\sigma_{E} / E \sim$ $50 \% / \sqrt{E(G e V)}$. The results of the experiment at Nuclotron with the use of ${ }^{6} \mathrm{Li}$ beam shown the similar energy resolution for nucleons [12. However, optimization of the module design for Nuclotron energies is required.

In this paper the results of the Monte-Carlo simulation on the optimization of the scintillator/lead hadronic calorimeter for Nuclotron energies as well as the systematic studies of the HCAL module for the peripheral part of ZDC with muon beam are presented.

\section{Monte-Carlo simulation for BM@N ZDC}
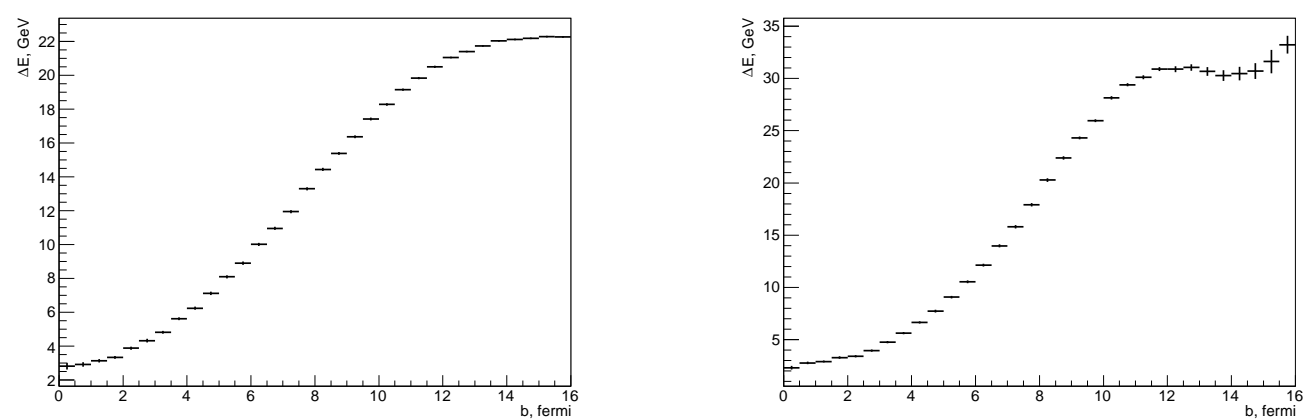

Figure 2: The total energy $\Delta E$ deposited in ZDC as a function of impact parameter $b$ for UrQMD [13] (left) and LAQGSM [14] minimal bias events at $4 \mathrm{GeV} /$ nucleon.

One of the main parameters of ZDC is the average part of the total spectators energy registered in the calorimeter related to the total spectators energy. With this part increasing the events classification by centrality appear more reliable. Other important ZDC parameter is the energy resolution. The provided error in the measurement of the total spectators energy should be less, than average fluctuations of the spectators energy for the central collisions. Thus, angular acceptance (actually cross-section size) and energy resolution should provide high efficiency of events selection on the basis of the centrality. 
Total energy deposited in ZDC

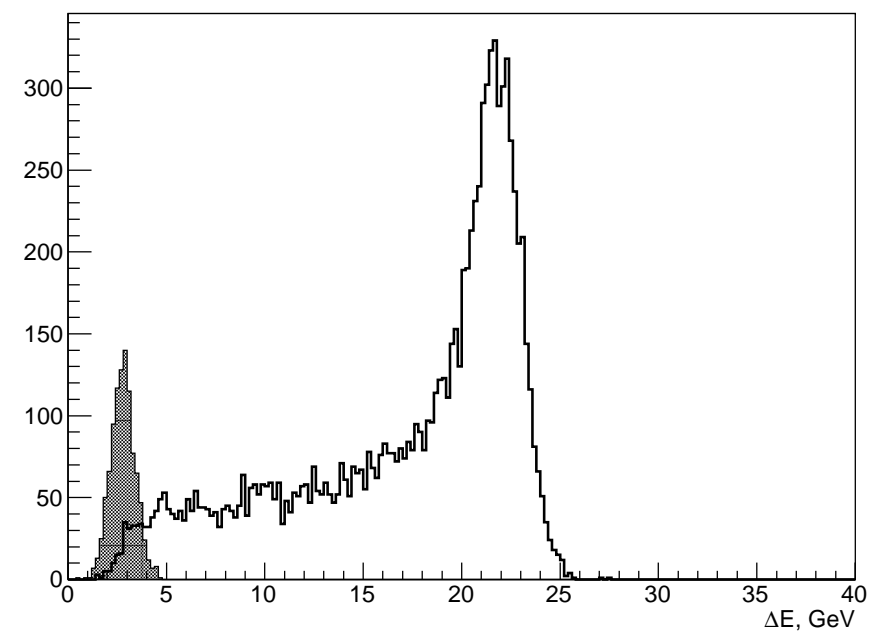

Figure 3: The energy deposition in ZDC for the central and minimum bias UrQMD [13] events shown by hatched and open histograms, respectively.

The simulation of the ZDC response has been performed using $\mathrm{Au}+\mathrm{Au}$ central and minimum bias UrQMD [13] and LAQGSM [14] events at 4.0 $\mathrm{GeV} /$ nucleon and BM@N software framework described in ref.[7]. The transverse size of ZDC was taken as shown in Fig 1. The position of ZDC was taken as $10 \mathrm{~m}$ from the target point. The fraction of the total energy deposited in ZDC is about 0.6 for the maximal magnetic field in the BM@N magnet. This fraction will increase with the magnetic field decreasing. The dependences of the deposited in ZDC total energy $\Delta E$ on the impact parameter $b$ for the minimum bias UrQMD [13] and LAQGSM [14] events are shown in the left and right panels of Fig. 2, respectively. The correlation of the total energy $\Delta E$ and impact parameter $b$ clearly seen in Fig. 2 allows to use ZDC information to estimate the centrality of the collisions. One can see, that there is no difference for the central and semi-central events for UrQMD [13] and LAQGSM [14] models. However, LAQGSM [14] gives larger energy deposition for the minimal bias events due to taking into account nuclear fragments.

The energy deposition in ZDC for the 1000 central and 10000 minimum bias UrQMD [13] events is shown in Fig. 3 by the hatched and open histograms, respectively. The average value of the deposited energy in ZDC is $\sim 3 \mathrm{GeV}$ and $\sim 22 \mathrm{GeV}$ for the central and minimum bias events, respectively. Therefore, the signal from ZDC can be used also for the trigger purposes to 
select the events within fixed range of the centrality.

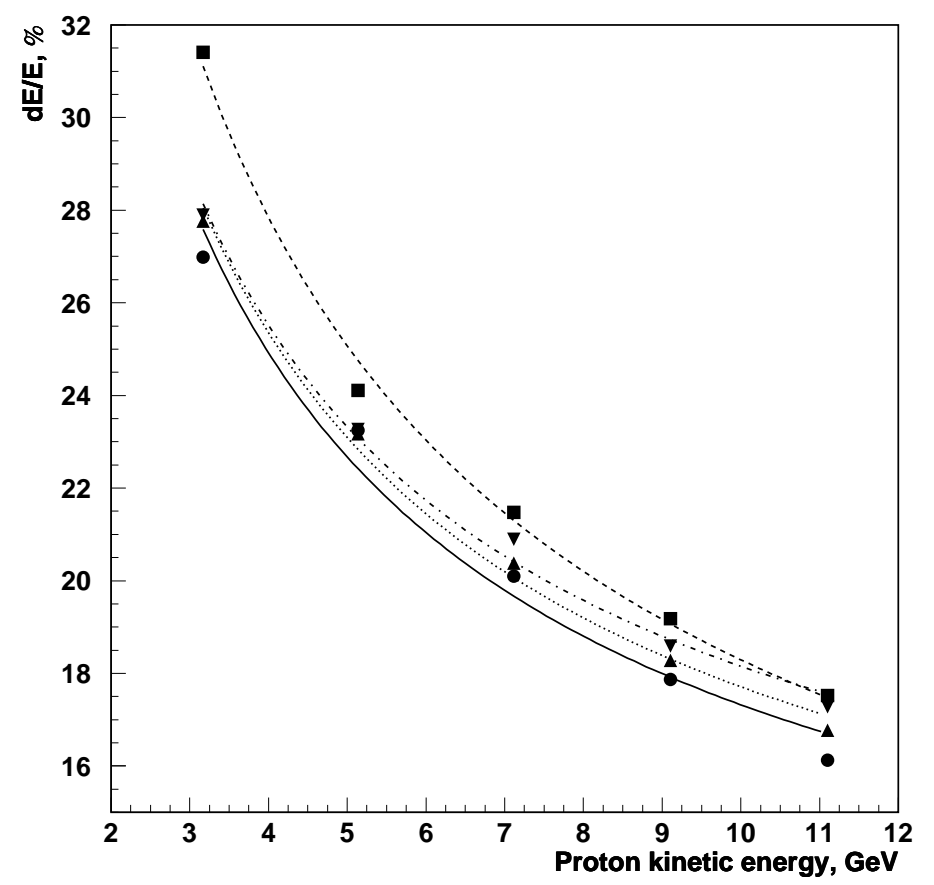

Figure 4: ZDC energy resolution $\sigma_{E} / E$ as a function of the proton kinetic energy $T$ for different scintillator/lead sampling structures. The symbols and lines corresponds to the following number of scintillator/lead layers $n$ and scintillator thickness $l_{s}$ : circles and solid line $-n=65$ and $l_{s}=4 \mathrm{~mm}$, triangles and dotted line $-n=65$ and $l_{s}=5 \mathrm{~mm}$, rotated triangles and dashdotted line $-n=49$ and $l_{s}=10 \mathrm{~mm}$, squares and dashed line $-n=78$ and $l_{s}=2.5 \mathrm{~mm}$, respectively. The lines are the results of the interpolation by the function $a / \sqrt{E(G e V)} \oplus b$.

ZDC energy resolution plays ZDC energy resolution $\sigma_{E} / E$ as a function of the proton kinetic energy $T$ has been studied for different calorimeter scintillator/lead sampling structures. The lead absorber plate thickness was fixed to $10 \mathrm{~mm}$, while the number of scintillator/lead layers $n$ and scintillator thickness $l_{s}$ were changed. The results for the $n=78$ and $l_{s}=2.5 \mathrm{~mm}$, $n=65$ and $l_{s}=4 \mathrm{~mm}, n=65$ and $l_{s}=5 \mathrm{~mm}, n=49$ and $l_{s}=10 \mathrm{~mm}$ are shown in Fig 4 by the solid squares, circles, triangles and rotated triangles, respectively. The lines are the results of the interpolation by the function 
$a / \sqrt{E(G e V)} \oplus b$. The results of the interpolation for different calorimeter scintillator/lead sampling structures are summarized in Table 1.

Table 1: The simulation results for ZDC energy resolution for different calorimeter scintillator/lead sampling structures. Here $n$ and $l_{s}$ are the number of scintillator/lead layers and scintillator thickness, respectively. The lead absorber plate thickness is fixed to $10 \mathrm{~mm}$. The parameters $a$ and $b$ are obtained from the interpolation of the $\sigma_{E} / E$ energy dependence presented in Fig 4 by the function $a / \sqrt{E(G e V)} \oplus b$.

\begin{tabular}{|c|c|c|}
\hline $\begin{array}{c}\text { Scintillator } / \\
\text { lead sampling }\end{array}$ & $\begin{array}{c}a, \\
{[\%]}\end{array}$ & $\begin{array}{c}b, \\
{[\%]}\end{array}$ \\
\hline$n=78, l_{s}=2.5 \mathrm{~mm}$ & 54.2 & 6.4 \\
$n=65, l_{s}=4 \mathrm{~mm}$ & 46.3 & 9.3 \\
$n=65, l_{s}=5 \mathrm{~mm}$ & 46.9 & 9.7 \\
$n=49, l_{s}=10 \mathrm{~mm}$ & 46.3 & 10.7 \\
\hline
\end{tabular}

The results indicate that the achieved energy resolution is better than $50 \% / \sqrt{E(G e V)}$ for the scintillator thickness $l_{s} \geq 4 \mathrm{~mm}$. However, the homogeneity (parameter $b$ ) decreases with the increasing of the scintillator plates thickness. One can see, that the sampling structures $n=65, l_{s}=4 \mathrm{~mm}$ or $n=65, l_{s}=5 \mathrm{~mm}$ provide optimal values of the $a$ and $b$ parameters in the considered energy range. These results were taken into account to design of the HCAL module prototype.

\section{$3 \quad$ ZDC module prototype}

The HCAL module design is shown in Fig 5 . The module transverse size is equal to $15 \times 15 \mathrm{~cm}^{2}$, iron housing length is equal $120 \mathrm{~cm}$ and total length with photo-multiplier tube (PMT) is equal $145 \mathrm{~cm}$. The module components are placed in the light protection box which is as well module housing. The HCAL module prototype sampling structure consists of 66 scintillator/lead layers with thickness $4 \mathrm{~mm}$ and $10 \mathrm{~mm}$, respectively. Module prototype absorption length is about 5 interaction nuclear lengths $\left(\lambda_{\text {int }}\right)$. In this case ZDC energy resolution for hadrons $2-30 \mathrm{GeV}$ is $\sigma_{E} / E=46.3 \% / \sqrt{E(G e V)}$ $\oplus 9.3 \%$ according to the Monte-Carlo simulation results shown in Fig,4

The light from scintillator plates is transmitted from one side with a fast wavelength shifter (WLS) to a PMT placed inside the $\mu$-metal screen and 


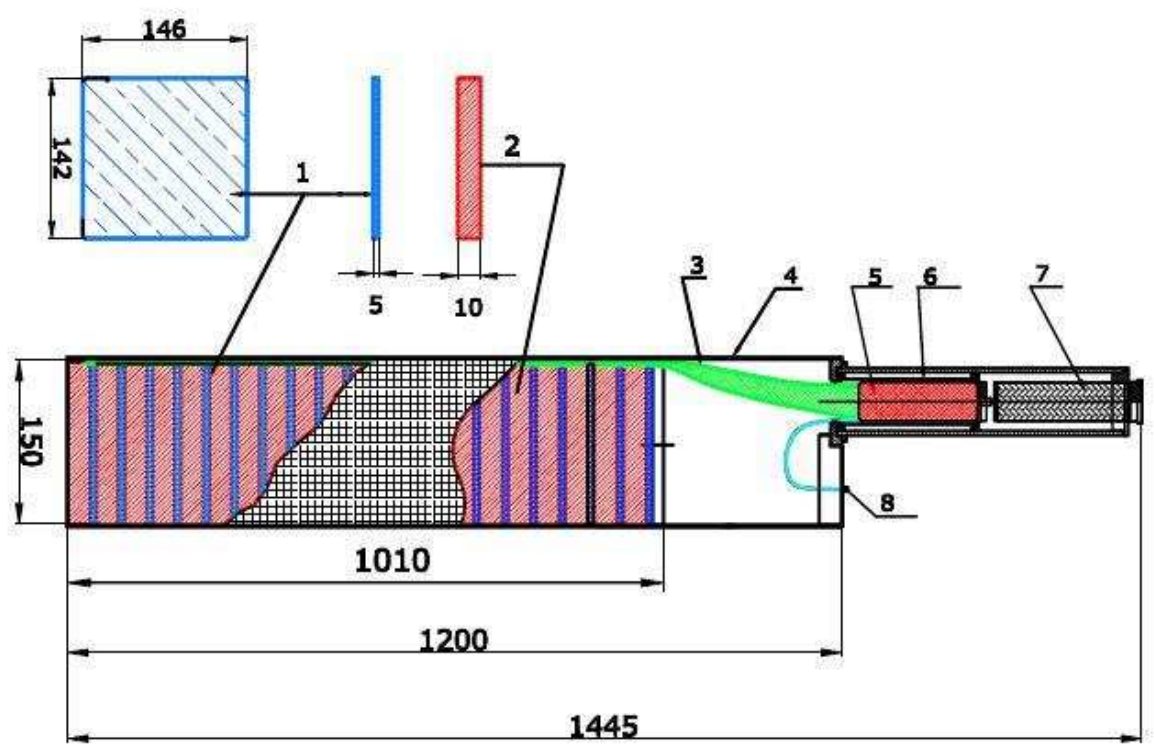

Figure 5: ZDC module prototype design. 1,2 - 66 scintillator/lead layers, 3 - WLS, 4 - iron housing, 5 - PMT, 6 - $\mu$-metal screen, 7 - PM high voltage base, 8 - fiber with an optical connector.

coupled with an high voltage base. The light emitted diode (LED) radiating a light with the wave length of $460 \mathrm{~nm}$ is used for the tests. The light from LED is transmitted to the photo-cathode of PMT via $1 \mathrm{~mm}$ diameter optical fiber and the optical connectors located at the rear side of the HCAL module prototype. The LED signal can be distributed among all calorimeter modules.

The parts of WA98 hadron calorimeter [10] will be used for assembling of BM@N ZDC [1]. Unfortunately, the light output from scintillators used in the WA98 calorimeter is decreased due to radiation damage. Also the light outputs for the scintillators taken from the centre and periphery of the WA98 calorimeter are different. The measurements were performed using light guide $20 \times 150 \mathrm{~mm}^{2}$ and FEU-85 PMT. The LeCroy-2249A ADC has been previously calibrated using photo-diode, the bin width is equals to 0.46 p.e. The light output is decreased for $\sim 20 \%$ and $\sim 40 \%$ for the periphery and centre of the WA98 ZDC [1].

Therefore, for the HCAL module prototype new scintillator plates were manufactured at IHEP using the method of the injection molding under pressure from the granulated polystyrene PSM-115. The additives composition 

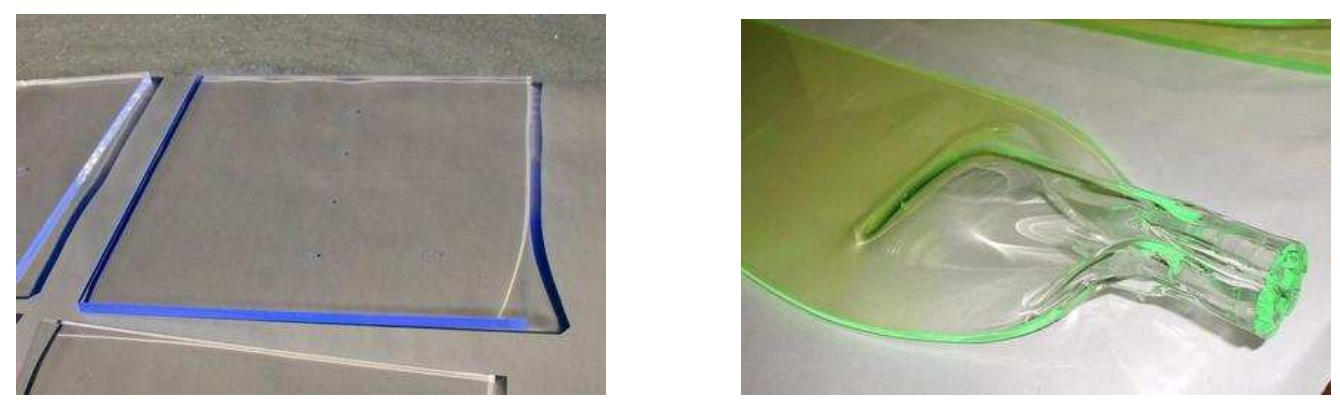

Figure 6: The components of the HCAL module prototype: scintillation plates (left) and light guide with wave length shifter (right).

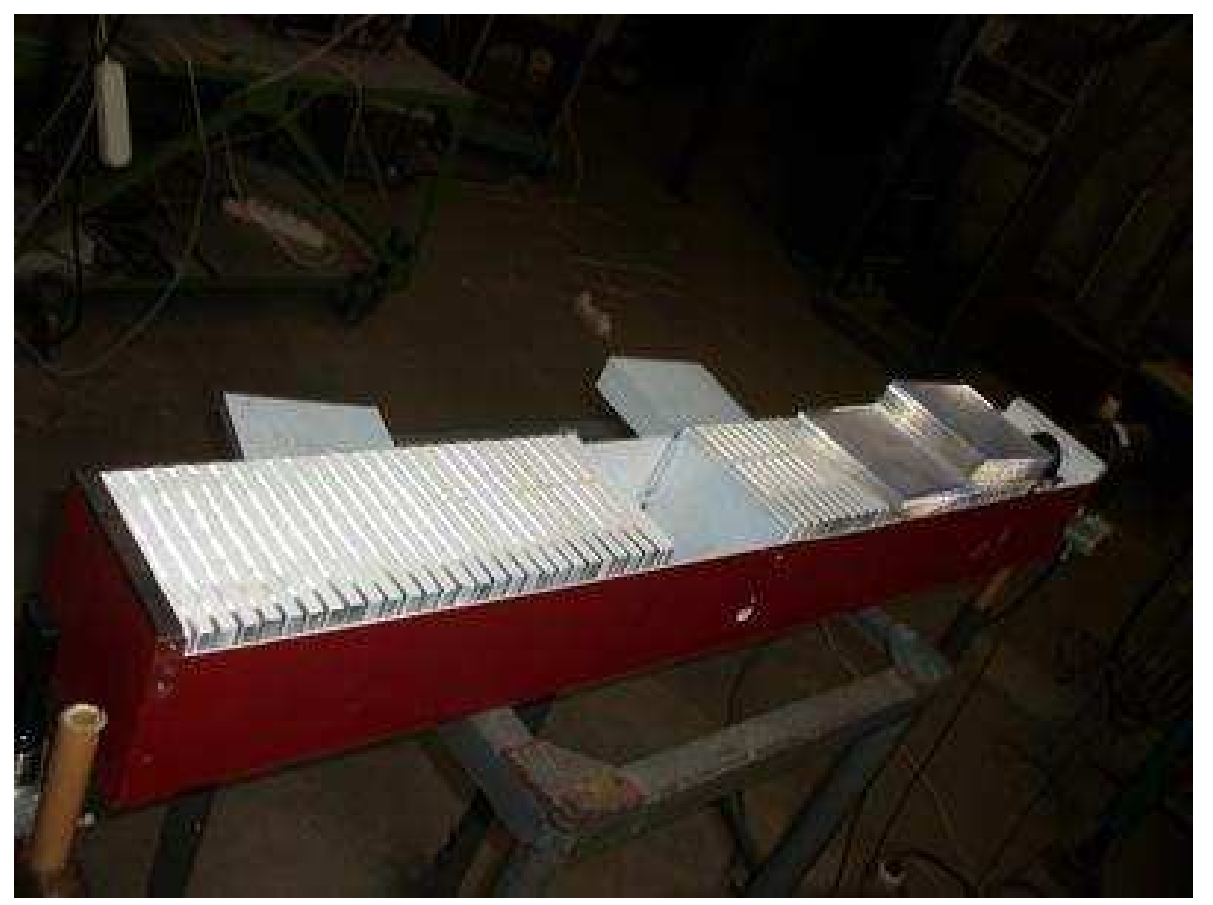

Figure 7: Inside view of the calorimeter module prototype. 
is P-terphenyl of $1.5 \%$ and POPOP of $0.05 \%$. Scintillator of this type have been successfully used in calorimeters for the last 20 years. It has a high radiation tolerance - about $10 \mathrm{MRad}$, a good light output (of $\sim 60 \%$ from the antracene light output), a fast decay time $\sim 1.5 \mathrm{~ns}$, a good transparency for blue light (wave length $\sim 420 \mathrm{~nm}$ ) with attenuation length of about 30-60 $\mathrm{cm}$.

The injection molding under pressure technology requires the use of the special matrix for scintillation plates production with given sizes. This matrix should be installed on a special injection machine with heating, extrusion and pressure systems. There is an cavity for the scintillator plates of given size at the centre of the matrix. The cavity shape have variable transverse size with the fixed thickness of $4 \mathrm{~mm}$. The use of this matrix allows to change the scintillation plates dimension up to $160 \times 300 \mathrm{~mm}^{2}$. The produced scintillator plates transfer size and thickness are equal to $142 \times 146 \mathrm{~mm}^{2}$ and $4 \mathrm{~mm}$, respectively. The matrix for the scintillator production as well as matrix with the injection machine are shown in Fig, 8 .
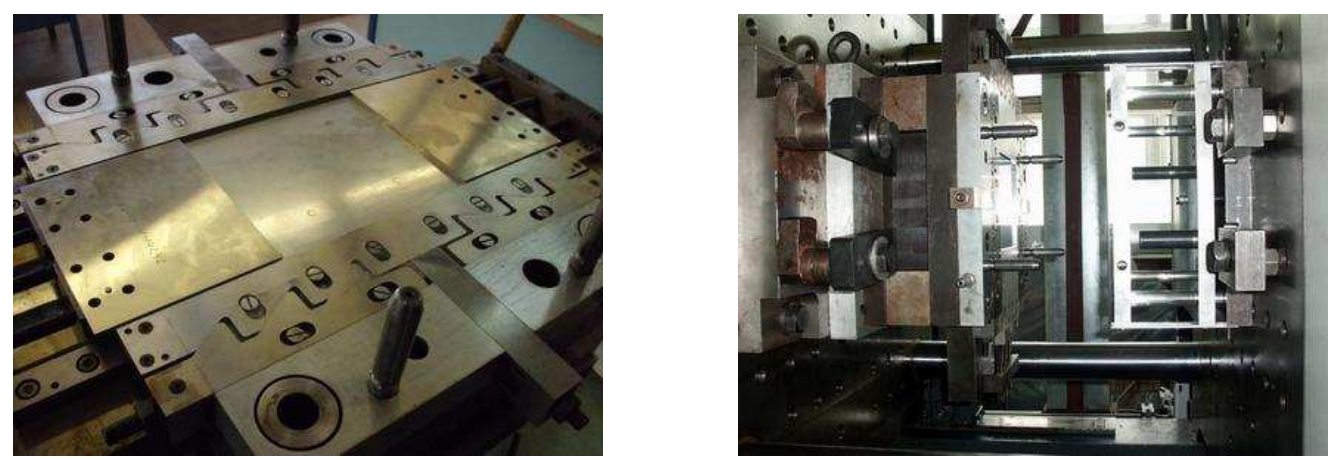

Figure 8: The matrix for the scintillator production (left) and and matrix installed on the injection machine (right).

WLS task is to absorb the blue light with a wave length $\sim 420 \mathrm{~nm}$ produced in the scintillator and radiate green light spectra with a wave length $\sim 520 \mathrm{~nm}$, which is optimal for the PMTs with bi-alkaline photocathodes. The WLS is manufactured from the optical organic glass (PMMA polymethylmethacrylate) with the surface painted by coumarin K-7 dissolved in the ethanol at $60^{\circ} \mathrm{C}$. The deepness of $\mathrm{K}-7$ diffusion has varied in range of 5-15 $\mu \mathrm{m}$. It allows one to obtain the good light collection uniformity along the WLS length $(1 \mathrm{~m})$ with the attenuation length larger than $3 \mathrm{~m}$. The WLS has a length of $120 \mathrm{~cm}$, width and thickness are equal to $12 \mathrm{~cm}$ and 0.4 $\mathrm{cm}$, respectively. The scintillation plates and light guide with wave length 
shifter used for the HCAL module prototype are shown in Fig 6 .

The absorber was made of lead plates $10 \mathrm{~mm}$ thick and $142 \times 146 \mathrm{~mm}^{2}$ transverse sizes. These lead plates were reused from WA98 hadron calorimeter [10]. The absorber was made from lead with $2 \%$ antimony to increase the hardness of the material. The light shielding box for module was made from steel sheets with a thickness of $1.5 \mathrm{~mm}$. Scintillation plates were not wrapped in reflective coating, while the lead plates and module housing inside were painted by white color to obtain diffusion light reflection. The mirror from Al-mylar was embedded at the bottom of the housing to reflect the light from the bottom edge. The inside view of the HCAL module prototype is shown in Fig.7.

\section{Beam test conditions.}

HCAL module prototype test was performed using muon beam with an energy of $\sim 10 \mathrm{GeV}$. The goal of the beam test was to measure the light yield and timing resolution for the minimum ionizing particles (MIPs) for the prototype for different types of PMT and WLS, as well as its transverse uniformity.

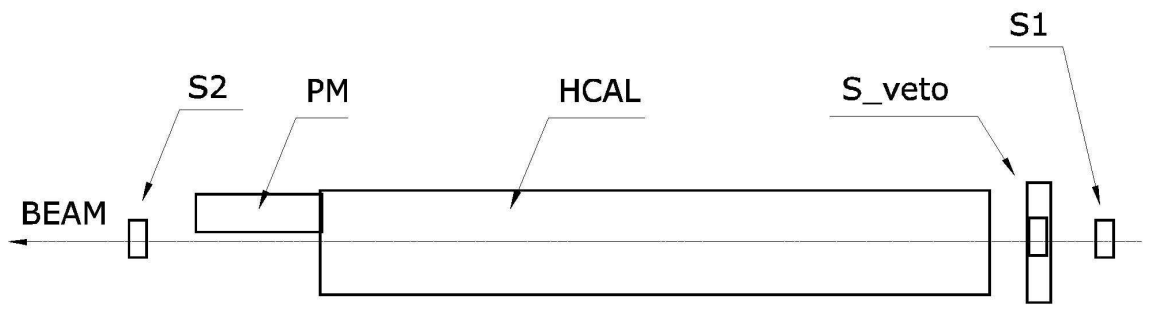

Figure 9: The layout of the beam test area. HCAL is the hadron calorimeter module prototype; $S_{1}, S_{2}, S_{\text {veto }}$ is the scintillation counters for trigger logic and definition the beam position, PM is the photomultiplier.

HCAL module prototype was installed in the beam test area as shown in Fig.9. Muons were generated by the secondary hadrons produced from the interaction of the proton beam with the internal nuclear target at accelerator U-70 and passed throughout the reinforced concrete protection. The muon beam has an energy $\sim 10 \mathrm{GeV}$ with intensity up to $10^{5} / \mathrm{cm}^{2}$ per spill. High energy muon beam passes through the HCAL module prototype parallel to its axis. 
The signals from three scintillation counters $S_{1}, \mathrm{~S}_{2}$ and $S_{\text {veto }}$ were used for the trigger purposes. The scintillators were coupled with the fast R3478 PMTs to obtain a good timing resolution of the trigger logic. Two of them, $S_{1}$ and $\mathrm{S}_{2}$, were installed in front and back of the prototype, respectively. Transverse dimension and thickness of their scintillators were $3 \times 3 \mathrm{~cm}^{2}$ and 2 $\mathrm{cm}$, respectively. The third counter $S_{\text {veto }}$ with the $3 \times 3 \mathrm{~cm}^{2}$ hole in the centre of the scintillator was also placed in front of the HCAL module prototype. The signals from PMT anodes were fed to a pulse shaper with constant threshold of $\sim 50 \mathrm{mV}$ through 50 ohms splitter. The signals coincidence from $S_{1}$ and $S_{2}$ counters defined the impact position and transverse size of the muon beam. The use of the $S_{\text {veto }}$ counter signal in the trigger logic allowed to avoid the multiple particles hitting in the module prototype. The LeCroy2249A and LeCroy2228A modules were used to store the charge and timing information from the scintillation counters and PMT of the HCAL module, respectively.
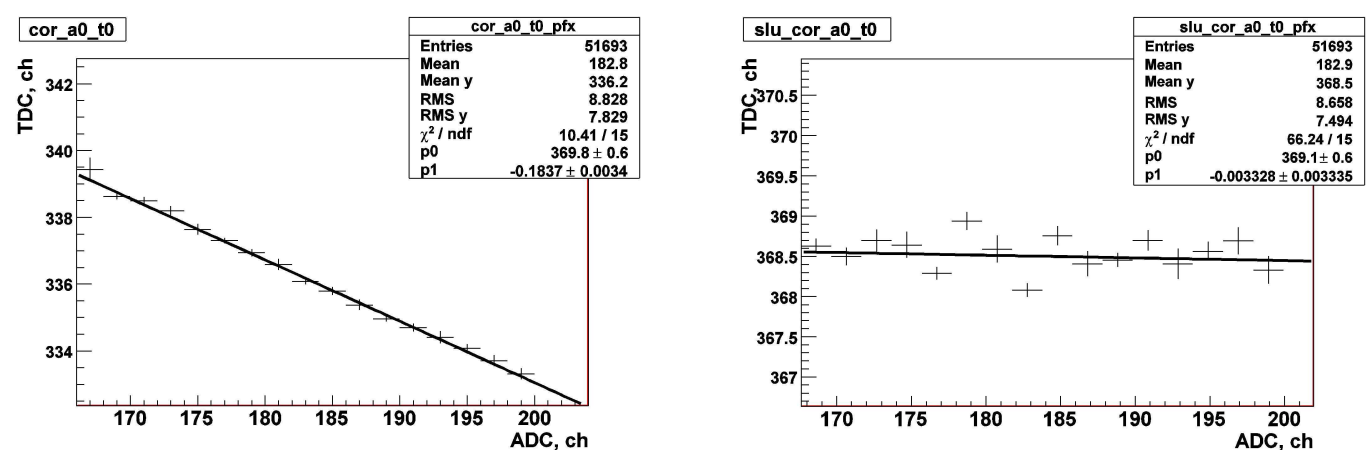

Figure 10: Time-amplitude dependence before (left) and after time-walk correction (right). Solid lines are the data fit by the linear functions.

The timing spectra for all PMT signals were corrected taking in account the time-amplitude dependence (so called time-walk effect seen in the left panel of Fig (10). This correction allow to compensate an amplitude time dependence as shown in the right panel of Fig.10. The final timing resolution for single trigger counter after correction was about 150 ps.

\section{$5 \quad$ Beam test results.}

The light output and timing resolution for different combinations of PMT and WLS in the HCAL module prototype have been measured with high energy 
muon beam. The reference measurements were performed using FEU-84 PMT and WLS of K-7 type. It was mentioned above that WLS of K-7 type has maximum in the emission spectrum at $520 \mathrm{~nm}$ and decay time about 6 ns. This WLS has a good uniformity and high conversion light output. FEU84 is a 12 stages PMT with bi-alkaline photocathode with diameter $25 \mathrm{~mm}$ and maximum of the spectral sensitivity at 420-460 nm. Such PMT-WLS combination was used previously in WA98 [10] and COMPASS [11 hadron calorimeters.
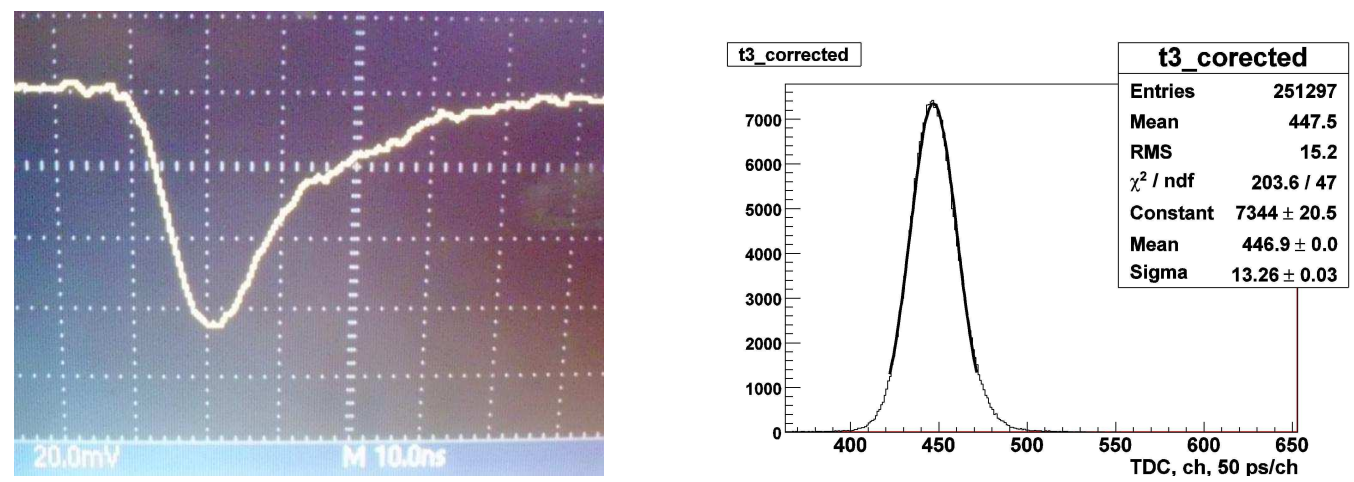

Figure 11: The signal pulse shape for FEU-84 PMT with WLS of K-7 type (left) and its timing spectrum (right) from the high energy muons. Solid line shows the data fit by the Gaussian function.

The signal pulse shape for FEU-84 PMT with WLS of K-7 type from the high energy muons passing through the middle of the HCAL module prototype is shown in the left panel of Fig.11. The pulse rise time and its full width at half maximum are $\sim 10 \mathrm{~ns}$ and $\sim 25 \mathrm{~ns}$, respectively. Timing resolution of the module prototype can be obtained from its timing spectrum presented in the right panel of Fig[11. It is equal to $\sim 640$ ps taking into account the trigger timing resolution of $150 \mathrm{ps}$.

EMI-9814KB is a 14 stage PMT with $46 \mathrm{~mm}$ bi-alkaline photocathode. The pulse rise time and its full width at half maximum obtained with high energy muons are $\sim 2 \mathrm{~ns}$ and $\sim 7 \mathrm{~ns}$, respectively, for the WLS of the K-7 type used. Timing resolution of the HCAL module prototype in this case is equal to $\sim 400$ ps taking into account the trigger timing resolution of 150 ps.

The HCAL module prototype amplitude spectra from the high energy muons for the FEU-84 and EMI-9814KB light detection are presented in the left and right panels of Fig,, 12 , respectively. The calibration the LeCroy2249A module charge scale was done using LED signal. The amplitude signal from 

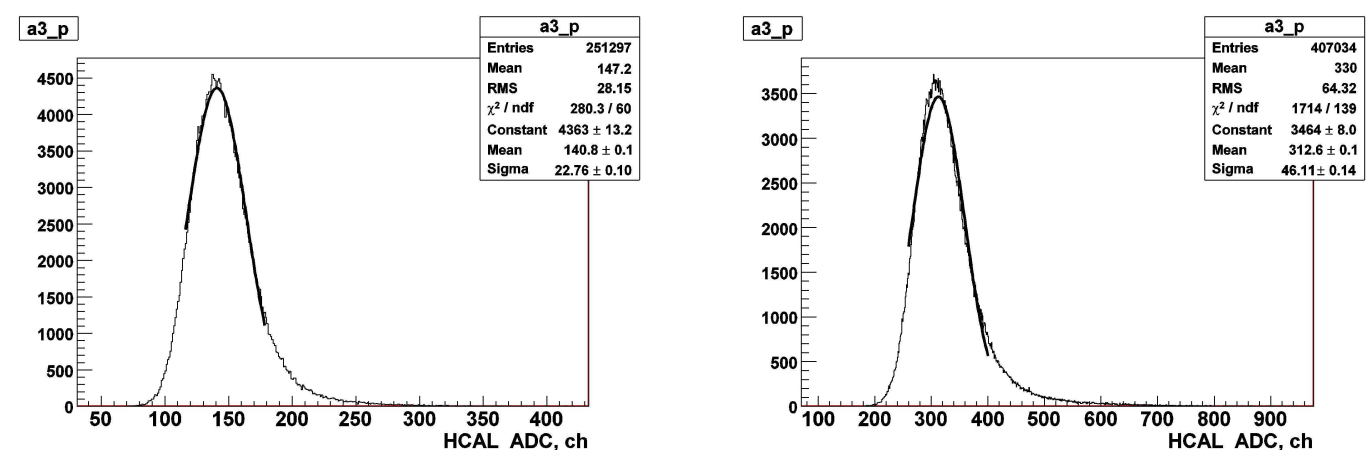

Figure 12: The HCAL module prototype amplitude spectra from the high energy muons for FEU-84 (left) and EMI-9814KB (right). The WLS of the K-7 type is used. Solid lines show the data fit by the Gaussian function.

the LED has been fitted by the Gaussian function. The number of photoelectrons $N_{\text {p.e. }}$ has been obtained as $N_{\text {p.e. }}=\left(\bar{A} / \sigma_{A}\right)^{2}$, where $\bar{A}$ and $\sigma_{A}$ are the mean value and standard deviation given by fit, respectively. The values of the calibration coefficients of $k=0.59 N_{\text {p.e. }} /$ channel and $k=0.49 N_{\text {p.e. }} /$ channel were obtained for FEU-84 and EMI-9814KB, respectively. The numbers of photoelectrons corresponding to the amplitude spectra obtained from muon beam and presented in Fig,12 are $N_{\text {p.e. }}=83$ and $N_{\text {p.e. }}=152$ for FEU-84 and EMI-9814KB, respectively. Therefore, the light output for EMI-9814KB is $\sim 1.8$ times larger than for FEU-84.

Table 2: The light output and timing resolution for HCAL module prototype for different PMT-WLS combinations.

\begin{tabular}{|c|c|c|c|}
\hline PMT type & WLS type & $\begin{array}{c}\text { Light output, } \\
N_{\text {p.e. }}\end{array}$ & $\begin{array}{c}\text { Timing resolution, } \\
\text { ps }\end{array}$ \\
\hline FEU-84 & K-7 & 83 & 640 \\
EMI-9814KB & K-7 & 152 & 400 \\
EMI-9814KB & K-30 & 108 & 408 \\
\hline
\end{tabular}

The dependence of the amplitude and timing response of the HCAL module prototype on the WLS type has been investigated with EMI-9814KB PMT. The similar timing resolution as for WLS of K-7 type has been obtained with WLS of K-30 type, namely, 408 ps. However, the light output is $\sim 1.4$ times lower than in the case of WLS of K-7 type use. Further optimization of the WLS painting procedure is necessary to obtain better light output 
with K-30 luminophore. The results of these measurements are presented in Table 2 .

Table 3: The normalized signal amplitude, time appearance and timing resolution for HCAL module prototype for different $X$ and $Y$ positions of the muon beam impact point. $X=0, Y=0$ is the prototype centre.

\begin{tabular}{|c|c|c|c|c|}
\hline $\begin{array}{c}X \text { - beam } \\
\text { position, } \\
\mathrm{mm}\end{array}$ & $\begin{array}{c}Y \text { - beam } \\
\text { position, } \\
\mathrm{mm}\end{array}$ & $\begin{array}{c}\text { Light } \\
\text { output } \bar{A}, \\
\%\end{array}$ & $\begin{array}{c}\text { Time } \\
\text { appearance } \Delta T, \\
\mathrm{ps}\end{array}$ & $\begin{array}{c}\text { Timing } \\
\text { resolution } \sigma_{T} \\
\mathrm{ps}\end{array}$ \\
\hline 0 & 40 & 100 & 0 & 410 \\
0 & -35 & 94 & 550 & 410 \\
35 & 40 & 105 & 50 & 410 \\
35 & -35 & 91 & 800 & 410 \\
\hline
\end{tabular}

Test of an uniformity of the HCAL module prototype was performed using muon beam directed to a given point of the prototype front side. PMT EMI-9814KB and WLS of K-7 type were used for the measurements. The direction of the muon beam was parallel to the module axis. The amplitude of the signal, its time appearance and timing resolution of the prototype were measured as a function of the muon beam impact point. The results of these measurements are presented in Table 3. Here $X=0$ and $Y=0$ correspond to the prototype centre. The signal amplitudes were normalized to the signal amplitude obtained for the muon beam hit in the impact point with the coordinates $X=0$ and $Y=40$. One can see that the normalized amplitude $\bar{A}$ decreases as the distance between the muon beam impact point and WLS is increased. The difference of the amplitudes for different coordinates of the muon beam impact point on the prototype reflects the light attenuation in the scintillation plates. Maximal amplitude changing is observed in the vertical direction as $\sim 10-15 \%$. Possible way to improve the light output uniformity of the module prototype could be the scintillator thickness increasing from $4 \mathrm{~mm}$ to $5 \mathrm{~mm}$. The Monte-Carlo simulation demonstrated that the energy resolution of the calorimeter will not change significantly in this case. The signal time appearance $\Delta T$ increases as the distance between the muon beam impact point and WLS is increased, while the timing resolution $\sigma_{T}$ was found to be $410 \mathrm{ps}$ independently on the muon beam impact point. The dependence of the $\Delta T$ on the distance from the hit position to WLS will play a role in the case of hadronic shower increasing the signal duration from single HCAL module. The observed uniformity of the timing resolution $\sigma_{T}$ 
across the section of HCAL module can indicate that there is no the ZDC energy resolution decreasing due to hit position dependence. However, the systematic studies of the HCAL module with hadron beam is required.

\section{Conclusions}

- The Monte-Carlo simulation results demonstrated the feasibility to use the proposed ZDC for the centrality determination in heavy-ion collisions for baryonic matter properties studies at Nuclotron energies.

- The results of Monte-Carlo simulation on the energy resolution of the hadron calorimeter in the energy domain of Nuclotron has been used to prepare HCAL module prototype.

- The response of the full scale prototype of the hadron calorimeter module for the peripheral part of BM@N ZDC to high-energy muons has been measured at U-70 accelerator for different combinations of PMT and WLS. The amplitude and timing spectra have been obtained.

- The results demonstrate that the use of EMI-9814KB PMT provides better timing resolution and $\sim 1.8$ times larger light output for the HCAL module prototype than for FEU- 84 PMT for WLS of K-7 type. The use of WLS of K-30 type with EMI-9814KB PMT shows also a good timing resolution. However, further optimization of the surface coating is required to increase light output as a whole.

- The non-uniformity of the light output due to attenuation in the scintillation plates was found $\sim 10-15 \%$. This value can be improved by the scintillator thickness increasing from $4 \mathrm{~mm}$ to $5 \mathrm{~mm}$.

The authors are grateful to Dr. A.Yu. Isupov for fruitful comments and remarks. They thank Prof. K.K. Gudima for the help in the simulation with LAQGSM generator.

\section{References}

[1] Ablyazimov T. O. et al. (BM@N Collaboration) // Conceptual Design Report of BM@N. http://nica.jinr.ru/files/BM@N/BMN-CDR.pdf 
[2] Kekelidze V. D., Sorin A. S. et al. (NICA Collaboration) // http://nica.jinr.ru

[3] Ladygin V. P. et al. (BM@N Collaboration) // PoS Baldin-ISHEPPXXI. 2012. P.038.

[4] Bratkovskaya E. et al.// Nucl. Phys. A. 2013. V.914. P.387.

[5] Vasiliev T. A., Ladygin V. P. and Malakhov A. I. // Nucl. Phys. Proc. Suppl. B. 2011. V.219-220. P.312.

[6] Kokoulina E. et al. (SVD-2 Collaboration) // PoS ICHEP2012. 2013. P.259.

[7] Ladygin V. P. et al.// In Proc. of the XV-th Advanced Research Workshop on High Energy Spin Physics (DSPIN-13), 8-12 October 2013, Dubna, Russia; Edited by A.V. Efremov and S.V. Goloskokov, JINR, Dubna, ISBN 978-5-9530-0315-3. 2014. P.239.

[8] Terekhin A. A. et al.// PoS Baldin-ISHEPP-XXI. 2012. P.005.

[9] Piyadin S. M. et al. // Phys.Part.Nucl.Lett. 2012. V.9. P.589.

[10] Arefiev V. A. et al.// JINR Rapid Communications. 1996. V.5[79]-96. P.15.

[11] Vlasov N. V. et al.// Instrum. Exp. Tech. 2006. V.49. P.41.

[12] Balandin V. P. et al.// JINR Preprint. R13-2008-196. 2008. Dubna. (in Russian).

[13] Bass S. A. et al.// Prog.Part.Nucl.Phys. 1998. V.41. P.225;

Bleicher M. et al.// J.Phys. G. 1999. V.25 P.1859.

[14] Toneev V. D. and Gudima K. K. // Nucl.Phys.A. 1983. V.400. P.173c; Toneev V. D., Amelin N. S., Gudima K. K. and Sivoklokov S. Yu. // Nucl.Phys.A. 1990. V.519. P.463c;

Amelin N. S., Staubo E. F., Csernai L. S. et al.// Phys.Rev.C. 1991. V.44. P.1541. 